\title{
Parvovirus B19 Myocarditis of Fulminant Evolution
}

\author{
Michael Spartalis ${ }^{\mathrm{a}, \mathrm{c}}$, Eleni Tzatzaki ${ }^{\mathrm{a}}$, Eleftherios Spartalis ${ }^{\mathrm{b}}$, Christos Damaskos $^{\mathrm{b}}$, \\ Sophie Mavrogeni ${ }^{\mathrm{a}}$, Vassilis Voudris ${ }^{\mathrm{a}}$
}

\begin{abstract}
Myocarditis is an inflammation of the myocardium. Clinical presentation ranges from non-specific systematic symptoms to fulminant collapse and sudden death. Sudden death occurs at rates of $8.6-12 \%$ and cardiomyopathy at $9 \%$. In active myocarditis, there is inflammatory cellular infiltrate with myocardial necrosis. The disease is distinguished by clinical presentation in fulminant and non-fulminant myocarditis. We present a rare case of a parvovirus B19-induced fulminant viral myocarditis in a young female. The patient presented with acute onset heart failure mimicking a myocardial infarction, followed by non-specific symptoms that had been misdiagnosed as urinary tract infection.
\end{abstract}

Keywords: Parvovirus; Fulminant myocarditis; Heart failure; Cardiac magnetic resonance

\section{Introduction}

Myocarditis is an inflammation of the myocardium. Clinical presentation ranges from non-specific systemic symptoms, asymptomatic electrocardiographic abnormalities, cardiogenic shock, fulminant heart failure, prodrome viral symptoms, masquerading acute coronary syndrome to sudden death [1]. The diagnostic evaluation consists of an electrocardiogram (ECG), cardiac biomarkers, endomyocardial biopsy (EMB), polymerase chain reaction (PCR) techniques and myocardial imaging [2]. Treatment choices in stable conditions are angiotensin-converting enzyme (ACE) inhibitors, b-blockers, aldosterone antagonists, interferon-a and -b. In unstable conditions, supportive care includes vasopressors, positive inotropic agents, diuretics, vasodilators and assist devices [3].

\section{Case Report}

A 17-year-old female patient with a past medical history sig-

Manuscript submitted July 23, 2017, accepted August 3, 2017

aDivision of Cardiology, Onassis Cardiac Surgery Center, Athens, Greece bLaboratory of Experimental Surgery and Surgical Research, University of Athens, Medical School, Athens, Greece

${ }^{\mathrm{c} C o r r e s p o n d i n g ~ A u t h o r: ~ M i c h a e l ~ S p a r t a l i s, ~ D i v i s i o n ~ o f ~ C a r d i o l o g y, ~ O n a s s i s ~}$ Cardiac Surgery Center, Fokidos 42, Athens 115 27, Greece.

Email: mspartalis@icloud.com

doi: https://doi.org/10.14740/cr580w nificant only for asthma presented to our emergency department with a main complaint of fatigue. Her mother noticed she had been having an unintentional weight loss of $10 \mathrm{~kg}$ over 2 weeks and brought her to a doctor who diagnosed her with (asymptomatic) urinary traction infection and treated her with sulfamethoxazole/trimethoprim (Smx-tmp). After 1 week, she developed fever, abdominal pain, nausea and vomiting and reached out for a second opinion.

A computed tomography (CT) scan of the abdomen and pelvis was ordered, and the results showed no abnormalities. She was discharged with second generation cephalosporin with the diagnosis of pyelonephritis.

After $24 \mathrm{~h}$, she returned to the emergency department hypotensive. The ECG showed ST elevation in leads I, II, aVL, $\mathrm{V}_{1}-\mathrm{V}_{6}$ and right bundle branch block (Fig. 1).

On arrival to the intensive care unit, she was hypotensive with a systolic blood pressure of $60 \mathrm{~mm} \mathrm{Hg}$ despite the administration of IV fluids. Dobutamine IV was initiated, and the blood work came up with cardiac troponin I (cTnI) $>50 \mathrm{ng} /$ $\mathrm{mL}$. Supportive therapy with inotropic drugs (dobutamine 10 $\mu \mathrm{g} / \mathrm{kg} / \mathrm{min}$ ) and an intra-aortic balloon pump were necessary to maintain sufficient cardiac output.

Echocardiography revealed severely impaired left ventricular systolic function with an ejection fraction of $20 \%$. Right ventricular function was normal with moderate to large pericardial effusion and moderate hemodynamic compromise.

The patient underwent urgent coronary angiography to exclude coronary artery disease, coronary vasospasm or spontaneously reperfused coronary occlusion. Coronary angiography revealed no coronary lesions or significant stenosis in the coronary vessels.

Due to diagnostic uncertainty, we opted for cardiac magnetic resonance (CMR). Magnetic resonance imaging detected inflammation and edema (Fig. 2) and revealed late gadolinium enhancement (LGE) in epicardial portions of the heart sparing of the subendocardium (Fig. 3) suggesting myocarditis from parvovirus B19 (mimicking ST elevated myocardial infarction).

Microbiological investigation of the serum tested positive for parvovirus B19 (552 copies $/ \mathrm{mL}$ ) without detection of other cardiotropic viruses. The patient was subjected to an EMB. The diagnosis was confirmed by a high myocardial parvovirus B19 load $(15,237$ copies/ $\mu$ g DNA) by PCR assay and the presence of positive signals in endothelial cells with parvovirus B19 immunostaining (Fig. 4). Intensive antiviral therapy with intravenous immunoglobulin and immunosuppressive therapy was initiated, and the patient remained stable and gradually 


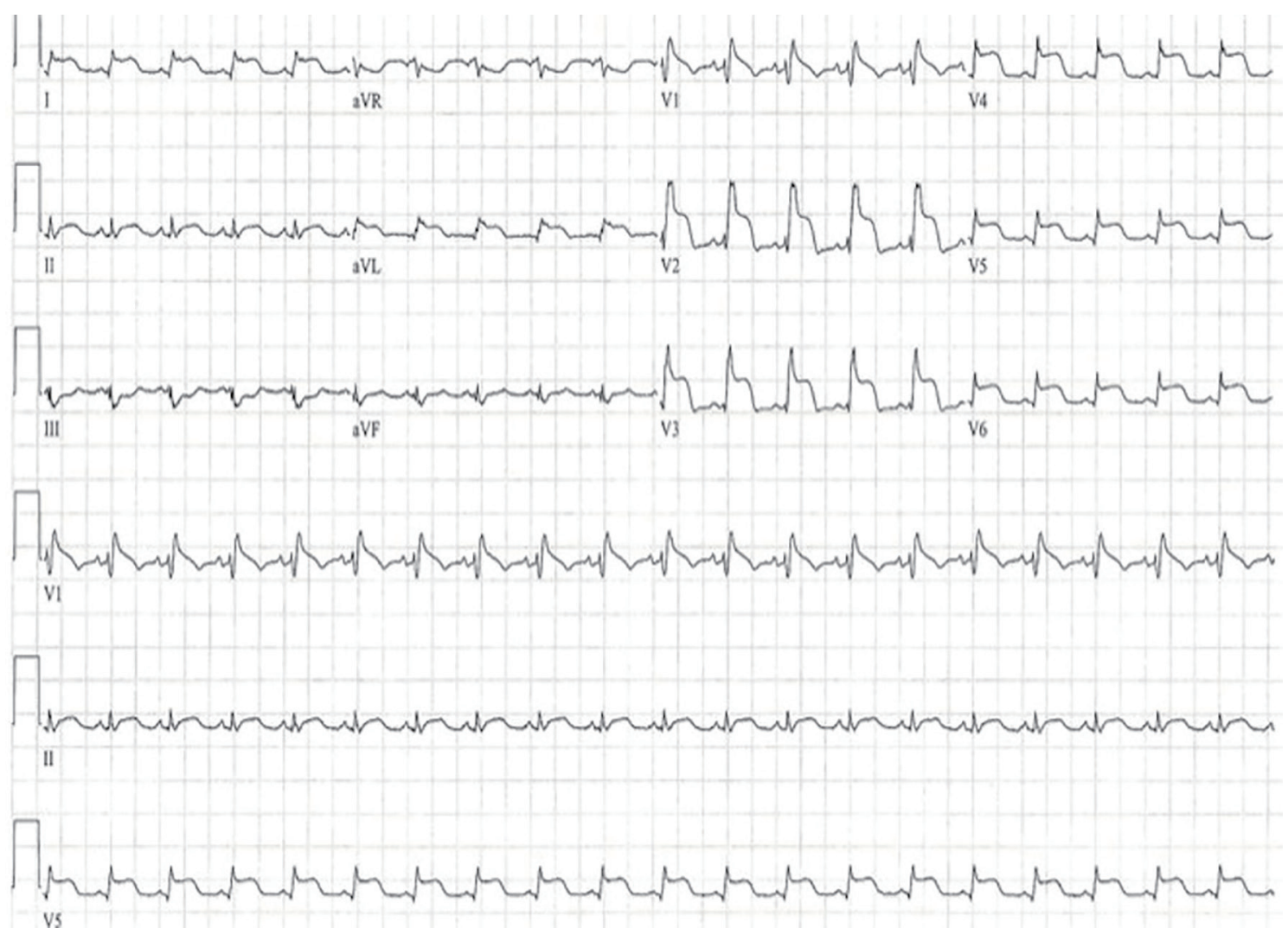

Figure 1. Twelve-lead electrocardiogram $25 \mathrm{~mm} / \mathrm{s}, 10 \mathrm{~mm} / \mathrm{mV}$, demonstrating persistent ST elevation in leads I, II, aVL, $\mathrm{V}_{1}-\mathrm{V}_{6}$ and right bundle branch block (RBBB).

recovered.

\section{Discussion}

This case emphasizes the utility of CMR in making a definite diagnosis of myocarditis in a patient with symptoms, signs, and an echocardiography examination suggestive of an ST-elevation myocardial infarction in the absence of coronary artery lesions. Approximately $10 \%$ of patients with typical angina symptoms and elevated troponin enzymes are found to have normal or non-significant coronary artery disease on angiography [4, 5]. Myocarditis etiology consists of infectious, autoimmune, or toxic factors, causing heart inflammation. The clinical spectrum of myocarditis is wide, ranging from the lack of symptoms to cardiogenic shock and sudden cardiac death. Myocarditis can also induce severe vasospasm mimicking acute myocardial infarction with acute onset of chest pain, echocardiogram abnormalities, and elevated cardiac enzymes $[1,2]$. This pattern of severe vasospasm is most frequently found in patients with biopsy-confirmed myocarditis from parvovirus B19. Increase in oxidative stress, reduced bioavailability of vasodilator NO availability and endothelial dysfunction have been suggested as possible mechanisms for induction of vasospasm $[4,5]$.

The clinical diagnosis of myocarditis can be challenging because of the often non-specific pattern of clinical presentation. Immunohistologic methods are more sensitive but still re- quire an EMB, which is highly invasive and not without risk. EMB is limited by sampling error ( $>17$ samples are necessary to diagnose myocarditis in $>80 \%$ of cases correctly) and is diagnostic only in 10-20\% of cases [3]. The current recommendations from the American Heart Association, the American College of Cardiology and the European Society of Cardiology (AHA/ACC/ESC) guidelines support a limited role for EMB in the evaluation of patients with cardiomyopathy [6]. The class I indications are limited to patients with new-onset heart failure $(<2$ weeks) associated with a normal or dilated left ventricle with hemodynamic compromise, moreover, to patients with new-onset heart failure of 2 weeks to 3 months duration with a dilated left ventricle, ventricular arrhythmia, or high degree atrioventricular block or to patients whose condition fails to respond to treatment in 1 - 2 weeks [6]. To the extent of re-evaluating the current guidelines, extensive research needs to be conducted in the setting of the role of EMB in the diagnosis of myocarditis. A biventricular EMB can provide additional value and become a useful tool. Different retrospective studies have suggested that biventricular EMB is superior in comparison to selective left ventricular or right ventricular biopsy [7].

CMR with the concomitant use of gadolinium not only provides valuable insights into structural and functional abnormalities of the heart but relevant tissue pathologies can also be visualized. The diagnostic accuracy is based on three tissue criteria (edema ratio, early gadolinium enhancement ratio and late gadolinium enhancement) $[8,9]$. CMR provides an assess- 


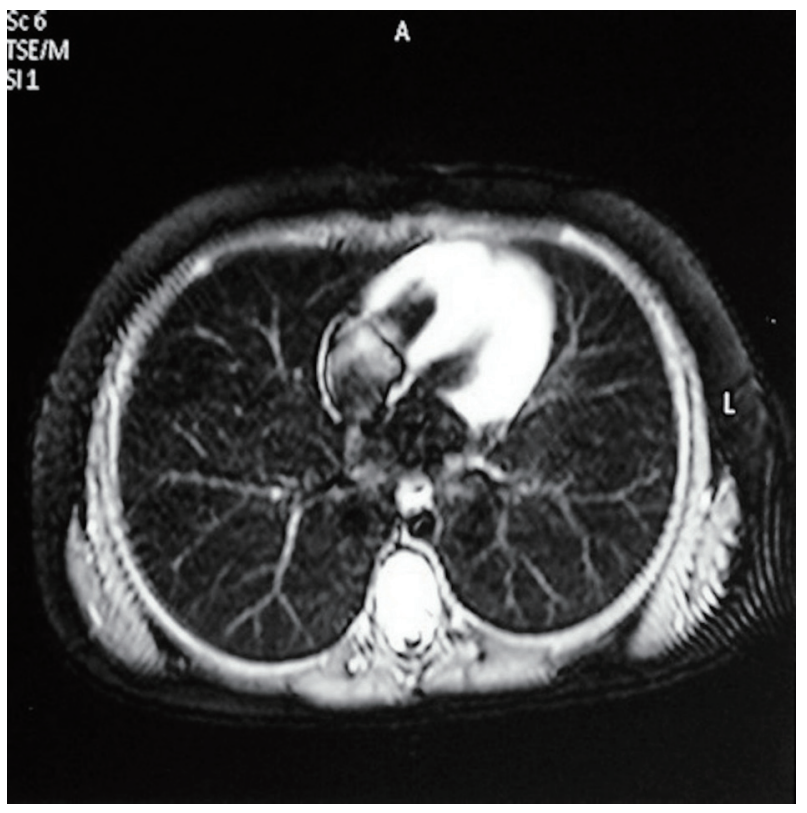

Figure 2. Cardiovascular magnetic resonance, T2-weighted, fourchamber view image revealing diffuse edema detected by increased signal integrity.

ment of myocardial edema, detection of inflammation, detailed evaluation of left and right ventricular function, "a noninvasive myocardial biopsy", detection of very small pericardial effusion and "a non-invasive follow-up" [8,9]. Mahrholdt et al demonstrated that LGE on approximately 10 min of gadolinium administration appears to correlate well with segments of histologically proven myocarditis [5, 9]. LGE locates in the mid-wall and tends to spare the subendocardium, which dif-

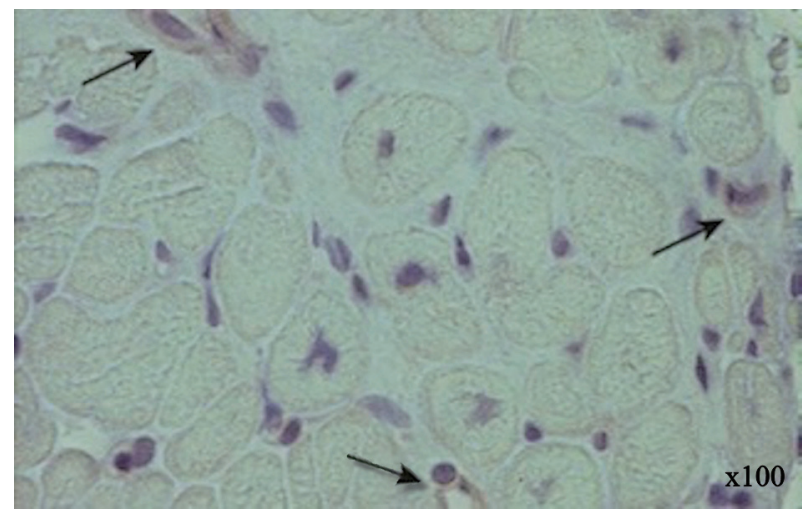

Figure 4. Parvovirus B19 immunostaining of endomyocardial biopsy sample showing positive parvovirus B19 immunohistology signals on endothelial cells (magnification $\times 100$ ).

ferentiates them from areas of infarction [5,9]. Mahrholdt et al also showed that the clinical presentation, the pattern of delayed enhancement on CMR, and the clinical course are correlated to the type of virus found in the myocardium [10]. Parvovirus B19-induced myocarditis tends to present commonly as severe acute recurring chest pain and is characteristically associated with LGE in the lateral wall [10]. In general, patients have a benign course with significant or complete recovery. In our case, the patient had a fulminant collapse differentiating our diagnosis from the typical parvovirus B19-induced myocarditis [4, 5].

The application of a standard CMR in fulminant myocarditis can clarify pathophysiology and also predict the evolution to heart failure. Mavrogeni et al suggested that the rapid development of massive edema contributes to rapid deterioration [11]. Large studies should be designed to evaluate the possible role of CMR in predicting the fulminant evolution of myocarditis.

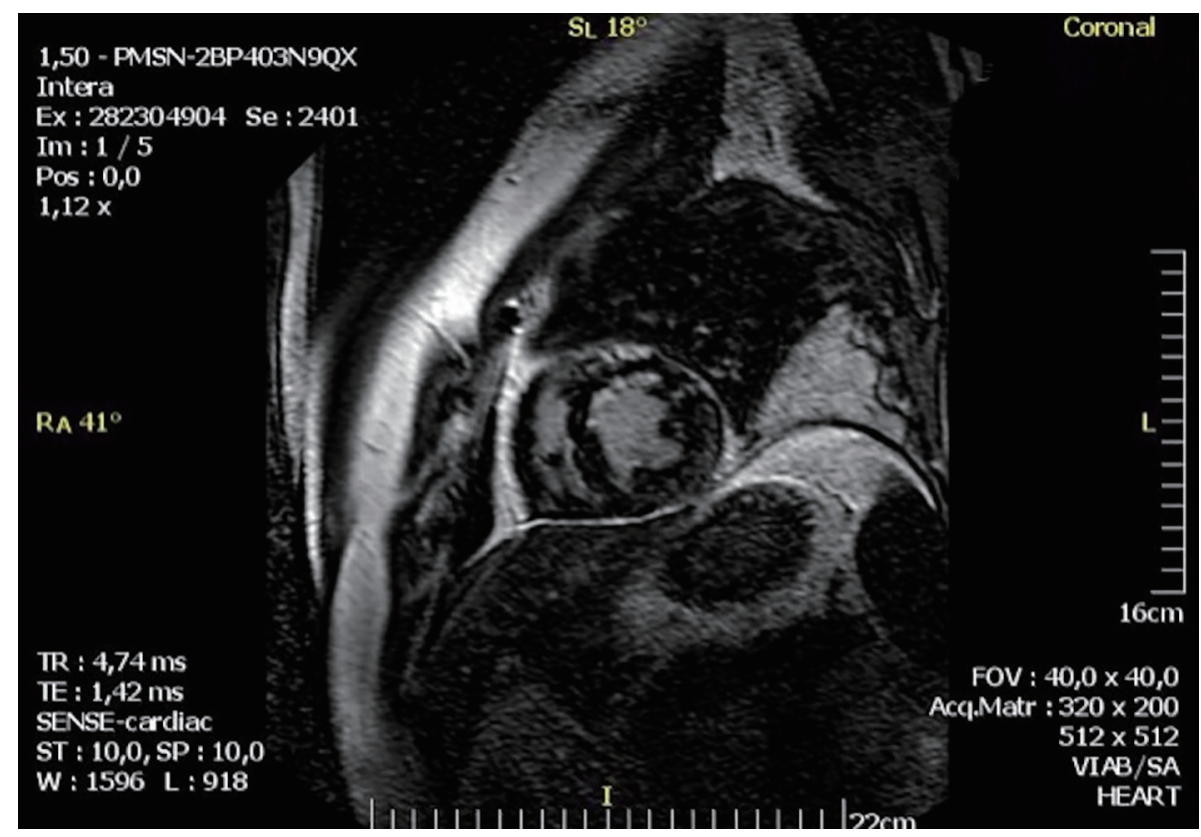

Figure 3. Cardiovascular magnetic resonance, T1-weighted, short axis view image revealing epicardial late gadolinium enhancement with sparing of the subendocardium. 
Treatment strategy regarding fulminant myocarditis consists of intense hemodynamic supportive therapy, which is proven to be most beneficial. Patients are often in cardiogenic shock and require intravenous inotropic agents and intra-aortic balloon pump for circulatory support. In case of persistent or worsening hemodynamic instability, a ventricular assistance device can be a bridge to recovery or even heart transplantation [1-3]. The role of additional immunosuppressive and antiviral therapy during the acute phase of fulminant myocarditis remains unclear and needs to be clarified.

\section{Conclusions}

This case illustrates a young female with a fulminant myocarditis associated with an active cardiac parvovirus B19 infection. The clinical presentation was typical of an ST-elevation myocardial infarction (STEMI). Cardiac magnetic resonance provided the early and prompt diagnosis. Hemodynamic support in conjunction with immunosuppressive and antiviral therapy resulted in complete recovery.

\section{Consent}

Written informed consent was obtained from the patient for publication of this case report and accompanying images.

\section{Financial Support}

This research received no specific grant from any funding agency, commercial or not-for-profit sectors.

\section{Conflicts of Interest}

All authors have contributed and approved the final version of this manuscript. No author has any conflicts of interest to disclose.

\section{References}

1. Sinagra G, Anzini M, Pereira NL, Bussani R, Finocchiaro
G, Bartunek J, Merlo M. Myocarditis in clinical practice. Mayo Clin Proc. 2016;91(9):1256-1266.

2. Fung G, Luo H, Qiu Y, Yang D, McManus B. Myocarditis. Circ Res. 2016;118(3):496-514.

3. Schultz JC, Hilliard AA, Cooper LT Jr, Rihal CS. Diagnosis and treatment of viral myocarditis. Mayo Clin Proc. 2009;84(11):1001-1009.

4. Basic D, Gupta S, Kwong RY. Parvovirus b19-induced myocarditis mimicking acute myocardial infarction: clarification of diagnosis by cardiac magnetic resonance imaging. Circulation. 2010;121(7):e40-42.

5. Mahrholdt H, Goedecke C, Wagner A, Meinhardt G, Athanasiadis A, Vogelsberg H, Fritz P, et al. Cardiovascular magnetic resonance assessment of human myocarditis: a comparison to histology and molecular pathology. Circulation. 2004;109(10):1250-1258.

6. Cooper LT, Baughman KL, Feldman AM, Frustaci A, Jessup M, Kuhl U, Levine GN, et al. The role of endomyocardial biopsy in the management of cardiovascular disease: a scientific statement from the American Heart Association, the American College of Cardiology, and the European Society of Cardiology. Endorsed by the Heart Failure Society of America and the Heart Failure Association of the European Society of Cardiology. J Am Coll Cardiol. 2007;50(19):1914-1931.

7. Stiermaier T, Fohrenbach F, Klingel K, Kandolf R, Boudriot E, Sandri M, Linke A, et al. Biventricular endomyocardial biopsy in patients with suspected myocarditis: Feasibility, complication rate and additional diagnostic value. Int J Cardiol. 2017;230:364-370.

8. Besler C, Schuler G, Lurz P. [Myocarditis in the differential diagnosis of cardiomyopathies. Endomyocardial biopsy or MRI?]. Herz. 2015;40(4):607-615.

9. Mahrholdt H, Wagner A, Judd RM, Sechtem U, Kim RJ. Delayed enhancement cardiovascular magnetic resonance assessment of non-ischaemic cardiomyopathies. Eur Heart J. 2005;26(15):1461-1474.

10. Mahrholdt H, Wagner A, Deluigi CC, Kispert E, Hager S, Meinhardt G, Vogelsberg H, et al. Presentation, patterns of myocardial damage, and clinical course of viral myocarditis. Circulation. 2006;114(15):1581-1590.

11. Mavrogeni S, Bratis K, Terrovitis J, Tsagalou E, Nanas J. Fulminant myocarditis. Can cardiac magnetic resonance predict evolution to heart failure? Int J Cardiol. 2012;159(2):e37-38. 